\title{
CHRONIC CONSTIPATION ENHANCES URINARY TRACT INFECTION IN CHILDREN: EXPERIENCES IN A TERTIARY CARE HOSPITAL OUTPATIENT DEPARTMENT
}

\author{
Amrita Lal Halder' ${ }^{1}$ M M Masud Pervez², Shareen Khan¹. \\ ${ }^{1}$ Department of Pediatrics and Neonatology, Bangladesh Institute of Research and Rehabilitation in \\ Diabetes, Endocrine and Metabolic disorders (BIRDEM) General Hospital, Dhaka, Bangladesh; \\ ${ }^{2}$ Department of Pediatric Surgery, Bangladesh Institute of Research and Rehabilitation in Diabetes, \\ Endocrine and Metabolic disorders (BIRDEM) General Hospital, Dhaka, Bangladesh.
}

\begin{abstract}
Background: Constipation and urinary tract infection (UTI) are two common pediatric problems. Chronic constipation is thought to enhance the occurrence of childhood UTI. So, prevention and treatment of constipation will reduce UTI and its complications such as renal scarring, hypertension and chronic renal failure. This study was conducted to find out the impact of chronic constipation on UTI in children.

Methods: This case-control study was done from August 2017 to December 2019 in the general pediatrics and pediatric surgery outpatient department (OPD) of a tertiary care children's hospital in Dhaka, Bangladesh. It was conducted on 164 children aged 1-15 years of which 82 constipated children who met Rome III criteria for chronic constipation were taken as case group and 82 non-constipated children as control group. Children having congenital urinary or anorectal anomalies detected by clinical examination and urogenital ultrasonogram (USG) were excluded from the study. Urine routine microscopic examination (RME) and culture-sensitivity (CS) were done for all patients. Growth of a single species of organism with colony count of $>10^{5} / \mathrm{ml}$ or colony counts $10^{4} / \mathrm{ml}$ with pyuria (pus cells $>5 / \mathrm{HPF}$ ) in the symptomatic child was considered as UTI.

Results: UTI was detected in 27 (32.9\%) constipated children and in 9 $(10.9 \%)$ non-constipated children $(p=0.0007)$. Within one month after proper treatment, 29 children underwent voiding cystourethrogram (VCUG); 24 in case group and 5 in control group. VUR was found in total 10 (34.5\%) children; $9(37.5 \%)$ in case group and $1(20 \%)$ in control group $(p=0.45)$.

Conclusion: Chronic constipation has significant impact on occurrence of UTI in children. So early and effective preventive measures and treatment for constipation will be useful to reduce occurrence of UTI.
\end{abstract}

\section{Introduction}

Constipation and urinary tract infection (UTI) are two common pediatric problems in daily outdoor practice. Both the conditions frequently remain underdiagnosed as symptoms are often mild until complicated. Functional constipation in children has a high prevalence (0.7\%-29\%) worldwide; both in developed and developing countries. ${ }^{1}$ In constipation, pressure from the loaded and distended rectum on the bladder neck causes obstruction to urine flow and

Address for Correspondance: Dr. Amrita Lal Halder, MBBS, FCPS; Assistant Professor and Resident Physician (Pediatrics), BIRDEM General Hospital-2 (Women and Children) Room - 224, 1st floor, 1/A Ibrahim Saroni, Segunbagicha, Dhaka-1000, Bangladesh.

Email: amrita_antak@yahoo.com

(C2021 Pediatric Oncall
ARTICLE HISTORY

Received 29 April 2021

Accepted 5 June 2021

\section{KEYWORDS}

Urinary tract infection (UTI), chronic constipation thereby impair bladder emptying. ${ }^{2}$ This mechanical effect also inhibits bladder by stimulation of detrusor stretch receptors which may cause vesicoureteral reflux (VUR) and UTI. ${ }^{3}$ Even sub-clinical UTI can lead to renal scarring, increasing the risk of hypertension and chronic renal failure (CRF). ${ }^{4}$ Children with recurrent UTI are noted to have large fecal reservoirs. ${ }^{5,6}$ Some children have asymptomatic bacteriuria, which is clinically silent or mild. But these children may have VUR in $11 \%$ cases of which $19 \%$ to $35 \%$ are school going children. ${ }^{7}$ Voiding dysfunction such as urinary incontinence and urinary retention are significantly increased in children with constipation. ${ }^{8}$ It thus appears that there is a correlation between the constipation and urinary incontinence, VUR and UTI. This study was conducted to find out the impact of chronic constipation on UTI in children.

Methods \& Materials

This case-control study was done from August 2017 
to December 2019 in the general pediatrics and pediatric surgery outpatient department (OPD) of BIRDEM General Hospital-2 (Women and Children), Dhaka, Bangladesh. This study was approved by institution review board (IRB). The sample size was calculated by non-probability and convenient method. Sick, unconscious, immunocompromised patients and children having congenital urinary or anorectal anomalies detected by clinical examination or urogenital ultrasonogram (USG) were excluded from the study. After exclusion of these patients a total of 82 patients aged 1-15 years met the Rome III criteria for chronic constipation ${ }^{9}$ during the study period that was taken as case group. Another 82 study subjects in the control group were selected randomly from the children who have attended the same OPD for a reason other than constipation and were matched according to gender and age. Informed written consent was taken from the parents or legal guardians of each study subject.

Data were collected in a data collection sheet from both the groups. History and examinations findings necessary for the study were recorded. Urine sample was collected aseptically and before any antibiotic therapy from all cases to do routine microscopic examination (RME) and culture-sensitivity (CS). To prevent contamination by periurethral flora, clean-catch midstream urine specimen was collected after proper washing of the genitalia. Urine samples were collected in a sterile pediatric urine collection bag in children who were below 2 years of age. Within half an hour of collection, CS was done in microbiology department of BIRDEM General Hospital. Pyuria, defined as pus cells >5/HPF in RME is supporting evidence of noncontamination of sample. ${ }^{4}$ Growth of a single species of organism with colony count of $>10^{5} / \mathrm{ml}$ in symptomatic or asymptomatic children was taken as significant evidence of UTI. However, colony count of $10^{4} / \mathrm{ml}$ for a single organism with associated pyuria in symptomatic children was also considered as UTI. ${ }^{10}$ History or presence of one or more symptoms including dysuria, frequency, hematuria, abdominal pain, flank pain and fever were considered as symptomatic UTI. ${ }^{11}$ Children of case group were advised to take foods with high fiber content. Advice on lifestyle modification of the children as well as family were given. Pharmacotherapy with lactulose was also given to all constipated children. Children diagnosed as UTI were treated with appropriate antibiotics for 7-14 days. USG of urinary system was done again for all diagnosed UTI patients to exclude any structural abnormality. They all were requested to do a voiding cystourethrogram (VCUG) within 1 month after complete treatment. They were also requested to follow-up in pediatric nephrology clinic for detection of recurrence of UTI and development of any complications. All recurrent UTI cases and cases having VUR were given an antibiotic prophylaxis as per institute's protocol.

\section{Statistical analysis}

Statistical analysis was performed using SPSS windows program version 19.0 (SPSS Institute, Inc., Chicago, IL, USA). Chi-square ( $x 2$ ) test was used for comparative analysis between two groups of study population. At $95 \%$ confidence limit $p$ value less than 0.05 was labeled as significant.

\section{Results}

Among 82 children, in the case group 39 were males $(47.6 \%)$ and 43 were females (52.4\%). On the other hand, the control group consisted of 44 males (53.7\%) and 38 females (46.3\%). Male: Female ratio was 0.9:1 in case group and 1.16:1 in control group showing no gender bias $(p=0.43)$. Most of the children, $61(74.4 \%)$ in case group and $53(64.6 \%)$ in control group, included in this study were below 5 years of age. Mean age was 2.7 years in case group and 2.4 years in control group, showing no significant difference between these two groups $(p>0.05)$. The most common age for constipation was between 1 to 5 years with the prevalence of $59 \%$ comparing to $8 \%$ in $10-15$ years. Prevalence of UTI was also high in 1-5 years of age in both case group (53\%) and control group (41\%). History of previous attack of UTI was found in 27 $(32.9 \%)$ patients in case group and $8(9.8 \%)$ patients in control group.

UTI as per case definition was seen in 27 (32.9\%) constipated children (case group) and in 9 (10.9\%) non-constipated children (control group) $(p=0.0007)$. Among the total UTI cases $(n=36), 22(61.1 \%)$ were girls and $14(38.9 \%)$ were boys $(p=0.10)$. VCUG was done within 1 month after treatment of both UTI and constipation. A total of 29 children underwent the procedure: 24 in case group and 5 in control group. Another 7 patients dropped out from the follow-up. VUR was found in total $10(34.5 \%)$ children; $9(37.5 \%)$ in case group and $1(20 \%)$ in control group $(p=0.45)$. Among the 10 VUR cases, 7 (70\%) had grade I (urine reflux into the non-dilated ureter) and $3(30 \%)$ had grade II (urine reflux into the pelvis and calyces without dilatation) VUR. The various organisms isolated in urine culture are depicted in Table 1 . Among the total 36 diagnosed UTI cases, 11 (30.6\%) patients came back with recurrent attack; 7 (63.6\%) patients had one and $4(36.4 \%)$ patients had two further attacks of UTI during the study period.

Table 1. Isolated organisms from urine culture $(\mathrm{N}=36)$

\begin{tabular}{lll}
\hline Organisms & $\begin{array}{l}\text { Case } \\
\text { Group } \\
\mathbf{n}(\%)\end{array}$ & $\begin{array}{l}\text { Control } \\
\text { Group } \\
\text { n (\%) }\end{array}$ \\
\hline Escherichia coli & $24(88.9 \%)$ & $5(55.6 \%)$ \\
Proteus mirabilis & $1(3.7)$ & $2(22.2)$ \\
Klebsiella pneumoniae & $2(7.4)$ & $0(0.0)$ \\
Total & 27 & 9 \\
\hline
\end{tabular}

\section{Discussion}

Chronic constipation (functional or organic) is one of the main risk factor for UTI. ${ }^{2}$ According to our study, the chance of occurrence of UTI is significantly more in patients who are constipated which is consistent with other studies. ${ }^{4,10}$ A study on 38 constipated children and 31 normal children as control reported that the prevalence of UTI was $42 \%$ and $19 \%$, respectively $(P<0.05) .{ }^{12}$ Association between constipation and UTI was also reported in many other studies. ${ }^{2,3,6,8,13,14,15}$ One study showed that constipated children were about 7 times more prone to develop UTI than those not 
constipated. ${ }^{2}$ Non-significance of sex ratio indicate that constipation is one of important risk factors of UTI regardless of gender which was more or less similar to the study of Loening-Baucke. ${ }^{15}$

Occurrence of both constipation and UTI were more in early age ( 1 to 5 years) in our study which is consistent with other study. ${ }^{4,10}$ History of recurrent attack of UTI is more common in constipated children as shown in our study $(32.9 \%$ vs $9.8 \%)$ is also observed in other study. $6,8,10$

In the present study the most isolated organism in urinary culture was Escherichia coli in both case and control group that are consistent with many other studies. $2,4,6,8,10,11,12,13,14,15$

Chronic constipation increases the incidence of VUR shown in many studies. $7,10,14$ Sarvari et.al showed $70 \%$ of patient undergoing VCUG after concomitant UTI and constipation had demonstrable VUR. ${ }^{10}$ In our study VUR was not significantly associated $(p=0.45)$; probably due to exclusion of all urogenital abnormal cases from the study. This was also the probable cause of absence of severe grade of VUR in our study.

Many other studies also showed improvement of UTI symptoms and prevention of recurrence after appropriate management of constipation. ${ }^{6,10}$ In our study high recurrence rate $(30.6 \%)$ was due to poor adherence with constipation management and presence of other risk factors for UTI. Proper treatment including laxatives for immediate relieve of constipation, lifestyle and dietary modification of patients and their families, prophylaxis in indicated cases are necessary to prevent recurrence of UTI and occurrence of VUR. ${ }^{3,6,7,8,10}$ Screening out of other risk factors for UTI and development of any complication (hypertension, CRF) are very essential.4,7,8 No such complication was found in any diagnosed UTI case in our study during the study period.

As multiple urine samples were not used for urine culture and morning urinary sample collection was not possible, these might reduce the chance of positive CS. A large sample size study along with multiple and morning urine sample may overcome the limitations.

\section{Conclusion}

Chronic constipation has significant impact on occurrence of UTI in children. So early and effective preventive measures and treatment for constipation will be useful to reduce occurrence of UTI.

\section{Compliance with Ethical Standards}

Funding: None

Conflict of Interest: None

\section{References:}

1. Whiting P, Westwood M, Watt I, Cooper J, Kleijnen J. Rapid tests and urine sampling techniques for the diagnosis of urinary tract infection (UTI) in children under five years: a systematic review. BMC pediatrics. 2005;5:4.

2. Sampaio $C$, Sousa AS, Fraga LGA, Veiga ML, Netto JMB, Barroso Jr U. Constipation and lower urinary tract dysfunction in children and adolescents: a populationbased study. Frontiers in pediatrics. 2016;4:101.

3. Yazbeck S, Schick E, O'Regan S. Relevance of constipation to enuresis, urinary tract infection and reflux. Eur Urol. 1987; 13:318-321.

4. Hoque SA, Islam MT, Ahmed F, Hanif M, Islam S, Matin M. Impact of Constipation in Children on Urinary Tract Infection (UTI). Bangladesh Journal of Child Health. 2010;34:17-20.

5. Blethyn A, Jones K, Newcombe R, Roberts G, Jenkins H. Radiological assessment of constipation. Arch Dis Child. 1995;73:532-533.

6. O'Regan S, Yazbeck S, Schick E. Constipation, bladder instability, urinary tract infection syndrome. Clin Nephrol. 1985;23:152-154.

7. Rushton $\mathrm{H}$. Urinary tract infections in children. Epidemiology, evaluation, and management. Pediatr Clin North Am. 1997;44:1133-1169.

8. Dohil R, Roberts E, Jones K, Jenkins H. Constipation and reversible urinary tract abnormalities. Arch Dis Child. 1994;70: 56-57.

9. Xin HW, Fang XC, Zhu LM, Xu T, Fei GJ, Wang ZF, et al. Diagnosis of functional constipation: agreement between Rome III and Rome II criteria and evaluation for the practicality. J Dig Dis. 2014;15:314-320.

10. Sarvari GH, Ghane Sherbaf F, Partovi S, Elmi S, Akhavan $H_{\text {, }}$ Bakhtiari E. The Relationship between Chronic Constipation and Urinary Tract Infection in Children: A Case-Control Clinical Study. Int J Pediatr. 2017;5:5715-5721.

11. Hodsone EM, Craig JC. Urinary Tract Infections in Children. In: Avner ED, Harmon WE, Niaudet P, Yoshikawa N, Emma F, Goldstein SL, editors. Pediatric Nephrology: Seventh Edition. Berlin Heidelberg: Springer; 2016:1696-1719.

12. Kasirga E, Akil İ, Yilmaz Ö, Polat M. Evaluation of voiding dysfunctions in children with chronic functional constipation. Turk J Pediatr. 2006;48:340-343

13. Inan $M$, Tokuc $B$, Aydiner $C Y$, Aksu B, Oner N, Basaran UN. Personal characteristics of enuretic children: an epidemiological study from South-East Europe. Urologia internationalis. 2008;81:47-53.

14. Halachmi S, Farhat WA. The impact of constipation on the urinary tract system. Int J Adolesc Med Health. 2008;20:17-22.

15. Loening-Baucke V. Urinary incontinence and urinary tract infection and their resolution with treatment of chronic constipation of childhood. Pediatrics. 1997;100: 228-232. 